\title{
Analysis of Effect of Eggshell Membrane Patching for Moderate-to-Large Traumatic Tympanic Membrane Perforation
}

\author{
Jong Yoon Jung ${ }^{1}$, Hee-Chul Yun ${ }^{1}$, Tae-Min Kim ${ }^{1}$, Jae Woo Joo ${ }^{1}$, In Sik Song ${ }^{1}$, \\ Yoon Chan Rah', Jiwon Chang ${ }^{2}$, Gi Jung $\mathrm{Im}^{1}$, and June Choi ${ }^{1}$ \\ ${ }^{1}$ Department of Otorhinolaryngology-Head and Neck Surgery, Korea University College of Medicine, Seoul, \\ ${ }^{2}$ Department of Otorhinolaryngology-Head and Neck Surgery, Hallym University College of Medicine, Seoul, Korea
}

\author{
Received June 2, 2016 \\ Revised August 19, 2016 \\ Accepted August 22, 2016
}

Background and Objectives: To evaluate the effect of eggshell membrane (ESM) patching for tympanic membrane (TM) perforation, and to investigate correlations between healing time and age, gender, patching time, perforation size, and perforation location. Subjects and Methods: One hundred and seventy-five patients diagnosed with traumatic TM perforation at the Department of Otorhinolaryngology-Head and Neck Surgery, Korea University Ansan Hospital, between January 2008 and October 2013. Patients were divided into two groups, according to perforation edge approximation or ESM patching treatment. Healing time was compared between the group that received perforation edge approximation and the group that received ESM patching. Perforation grade, age, onset, and location were also compared between the two groups. Results: ESM patching significantly improved healing time compared to spontaneous healing, especially in patients with moderate or large traumatic TM perforations ( $\geq$ grade II). However, patient age, gender, perforation location, and especially timing of procedure, did not significantly affect healing time. Conclusions: ESM patching can be a good treatment choice to promote tympanic membrane healing in large traumatic TM perforations.

J Audiol Otol 2017;21(1):39-43

\section{Introduction}

The tympanic membrane (TM) divides the external ear and the middle ear. Its function is to perceive sound, and it is greatly sensitive to changes in external auditory canal air pressure. Sudden powerful forces to the ear, such as may be caused by hand, object, or car accident, can cause increased ear canal air pressure. Traumatic TM perforation is routinely observed in our otolaryngology outpatient clinic.

It is well established that most traumatic TM perforations tend to close spontaneously (76-94\%) [1-6]. However, those that fail to heal can result in moderate-to-large perforations. A majority of otolaryngologists agree that performing early

This is an Open Access article distributed under the terms of the Creative Commons Attribution Non-Commercial License (http://creativecommons.org/licenses/by-nc/4.0/) which permits unrestricted non-commercial use, distribution, and reproduction in any medium, provided the original work is properly cited. myringoplasty can improve healing rate $[7,8]$. However, this procedure involves risk, cost, and the inconvenience of an operation. Therefore, a non-surgical procedure, such as patch application, is usually selected as initial treatment. Various patching materials have been proposed, including paper, silk, gelatin sponge, fibroblast growth factor, insulin-like growth factor, chitosan, and human urinary bladder. However, the benefits and duration of each material are controversial [1,9].

Eggshell membrane (ESM), routinely used in wound dressings, is another potential patching materials [10]. In a previous study, we reported that the healing ratio of the TM was not significantly different between the ESM patch group and the edge approximation group, but that healing time was significantly shorter in the ESM patch group [10]. The present study had two main objectives: 1) to investigate the effect of ESM patching of traumatic TM perforation, according to the Griffin's TM perforation grade, and 2) to compare healing 
time according to the patching time, perforation location, and age in the ESM patch application group.

\section{Subjects and Methods}

\section{Data source and study population}

This is a retrospective review of 175 patients diagnosed with traumatic TM perforation at the Department of Otorhinolaryngology-Head and Neck Surgery, Korea University Ansan Hospital, between January 2008 and October 2013. Exclusion criteria were as follows; patients with temporal bone fracture, disruption of ossicles, previous perforation, or incomplete closure.

The external auditory canals of all 175 ears were cleaned when the patients visited the hospital, and the location and size of the perforation were recorded by ear endoscopy. Pure tone audiometry was performed before treatment.

TM perforation size was evaluated by Griffin's criteria [11] and graded into four categories, as follows, grade I=perforation involved $\leq 25 \%$ of the pars tensa, grade $\mathrm{II}=$ perforation involved $25-50 \%$ of the pars tensa, or multiple perforations involved two quadrants, grade III=perforation involved 50$75 \%$ of the pars tensa, or multiple perforations involved three quadrants, and grade IV=perforation involved $75-100 \%$ of the pars tensa [11]. The study was approved by the institutional review board of Korea University College of Me-dicine, and conducted according to the principles expressed in the Declaration of Helsinki (AS10131-001).

\section{Treatment selection}

ESM patch $(n=111)$ or approximation $(n=64)$ was randomly selected for treatment of the traumatic TM perforation by the patients physician. Oral antibiotics were administered for 1 week to prevent infections. Patients were regularly followed-up at the outpatient clinic until the TM perforation was closed. Perforation closure was confirmed by endoscopic examination.

\section{ESM patch group}

The eggs used for ESM patches were obtained from a traditional market. Eggs were washed with distilled water and brushed with a nylon brush. Eggs were then boiled in distilled water for $5 \mathrm{~min}$, broken, and the egg content was emptied. The remaining hard eggshell with membrane was washed with distilled water and the membranes were carefully extracted by hand. The extracted ESM was cut to make a round disc with a skin puncture. The final ESM patch diameter was $5 \mathrm{~mm}$. ESM patches were sterilised with ethylene gas [10]. During the ESM patch application, flaps of the perforation were approximated where possible and the ESM patch was placed in saline for moistening before application. The operator placed the ESM patch on the lateral surface of the perforation with the guidance of a microscope, and then ensured that it adhered to the TM [10]. The ESM patch was retried to 3 times during 3 months. After the ESM patch was removed spontaneously, tympanic membrane was evaluated.

\section{Approximation group}

Under an otomicroscope, EMLA $^{\circledR}$ cream (lidocaine 2.5\% and prilocaine $2.5 \%$; Astrazeneca AB, Karlskoga, Sweden) were applied into the EAC for 10 minutes. The inverted edges were aligned back to the original position as much as possible using a Rosen needle or a vacuum suction tip, however no patching materials were used.

\section{Statistical analysis}

All data are expressed as mean \pm standard deviation (SD). We used independent T-tests for healing time according to treatment method, age, and time. In addition, we performed analysis of variance (ANOVA) for comparing healing time according to location of TM perforation using SPSS software version 21 (IBM SPSS Inc., Chicago, IL, USA). Differences were considered statistically significant when $p<0.05$.

\section{Results}

In the 175 ears with traumatic TM perforation, 111 (62.6\%) were to the left ear and $64(37.4 \%)$ were to the right ear. Mean

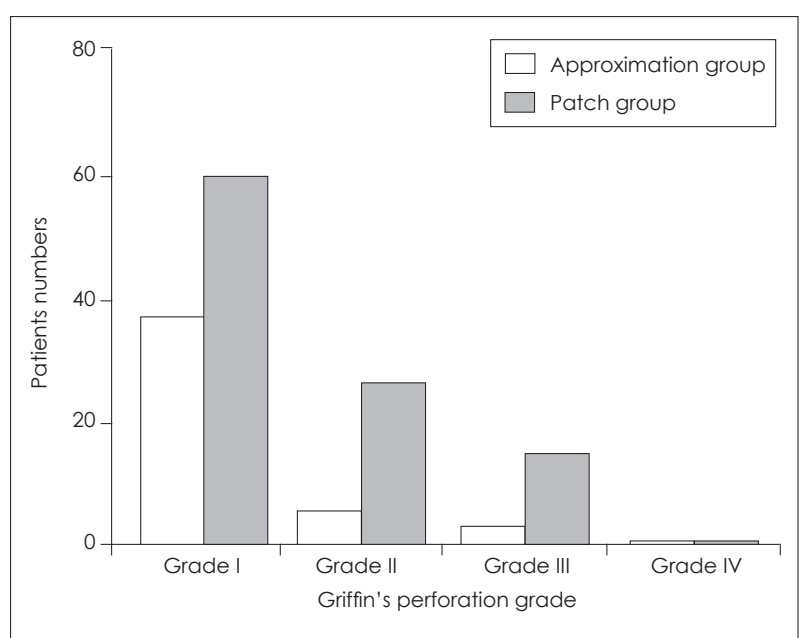

Fig. 1. Distribution of two groups according to Griffin's grade. There were 41 approximation patients and 62 ESM patch application patients with grade I perforation, 17 approximation patients and 32 ESM patch application patients with grade II perforation, 5 approximation patients and 16 ESM patch application patients with grade III perforation, and 1 approximation patient and 1 ESM patch application patient with grade IV perforation. ESM: eggshell membrane. 
patient age was $27.6 \pm 16.6$ years and the mean air-bone gap was $16.2 \pm 10.1 \mathrm{~dB}$. The number of patients with grade I perforation was 103, grade II was 49, grade III was 21 and grade IV was 2, as shown in Fig. 1.

Healing time analysis between the ESM patch group and the approximation group included 103 patients with small traumatic TM perforation (grade I) and 72 patients with moderate-to-large traumatic TM perforation ( $\geq$ grade II). Characteristics of the two groups are compared in Table 1. The mean perforation closure times were $37.3 \pm 24.5$ in the approximation group and $36.4 \pm 26.4$ days in the ESM patch group in patients with small traumatic TM perforation. Complete closure time was not statistically different between the 2 groups $(p=0.205)$. However, in patients with moderate-tolarge traumatic TM perforation, the mean perforation closure times was $110.4 \pm 127.4$ in the approximation group and 77.4 \pm 78.6 days in the ESM patch group. Healing time was significantly shorter in the ESM patch group than in the approximation group in patients with moderate-to-large traumatic TM perforation ( $p=0.007$ ) (Fig. 2).

TM closure time was analysed in 111 patients with ESM patching for TM perforation, according to age, patching time, and perforation location. The mean TM closure time was $58.5 \pm 23.9$ days for non-adult patients ( $\leq 18$ years) and 56.4 \pm 14.7 days for adult patients ( $>18$ years). There was no significant difference in TM closure time between non-adult patients and adult patients ( $p=0.591$ ) (Fig. 3).

The mean TM closure time was $54.8 \pm 34.7$ days for early patching patients ( $<1$ week post injury) and $62.3 \pm 39.6$ days for late patching patients ( $\geq 1$ week post injury, $p=0.326$ ). In the small TM perforation patients, there was no significant

Table 1. Summary of patient characteristics in the approximation group and the ESM patch group

\begin{tabular}{lccc}
\hline Characteristics & $\begin{array}{c}\text { Approximation } \\
\text { group }\end{array}$ & $\begin{array}{c}\text { ESM patch } \\
\text { group }\end{array}$ & p-value \\
\hline $\begin{array}{l}\text { Sex } \\
\text { (men:women) }\end{array}$ & $40: 24$ & $61: 50$ & 0.32 \\
$\begin{array}{l}\text { Site (left:right) } \\
\text { Mean age }\end{array}$ & $33: 31$ & $77: 34$ & 0.17 \\
$\quad$ (years) \pm SD & $25.91 \pm 17.97$ & $28.88 \pm 14.73$ & 0.16 \\
Patching time & $5.95 \pm 11.83$ & $4.22 \pm 7.19$ & 0.59 \\
$\quad$ (days) \pm SD & & & \\
Perforation grade & & & 0.30 \\
(no. of patients) & & 62 & \\
I & 41 & 32 & \\
II & 17 & 16 & \\
III & 5 & 1 & \\
IV & 1 & & \\
\hline
\end{tabular}

There was no significant difference of characteristics between approximation group and ESM patch group. ESM: eggshell membrane (independent T-test) difference in healing time between the early patching group and the late patching group ( $p=0.419$ ) (Fig. 4). The mean TM closure time was $54.9 \pm 21.6$ days for patients with anterior inferior quadrant TM perforation, $63.4 \pm 42.3$ days for patients with anterior superior quadrant TM perforation, 55.3 \pm 24.2 days for patients with posterior inferior quadrant TM perforation, and $70.2 \pm 76.5$ days for patients with posterior superior quadrant TM perforation $(p=0.252)$ (Fig. 5).

\section{Discussion}

Traumatic TM perforation is commonly observed in our otolaryngology outpatient clinics. Ear fullness, conductive hearing loss, middle ear infection, continued inflammation, and further tissue damage may result from continuous TM perforation $[4,11,12]$. Fortunately, most traumatic perforations heal spontaneously [1-6]. However, failure of complete closure and delayed TM perforation closure can occur in some cases. It has been suggested that curled edges of the perforated site edge cause the failure of complete spontaneous healing $[1,2,6]$.

In our previous study, a patch-applied group was com-

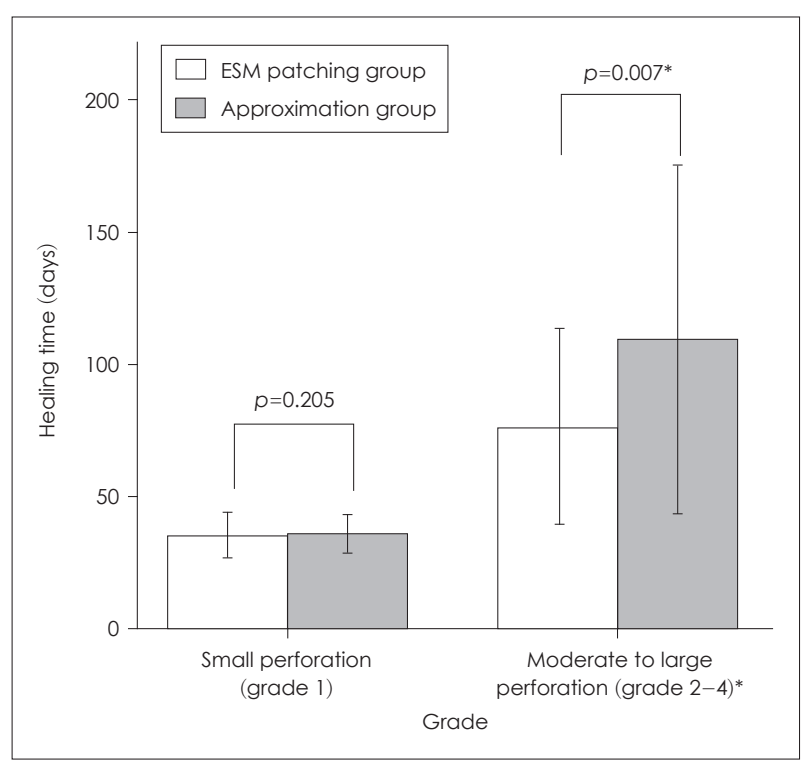

Fig. 2. Healing time in the ESM patching group and the approximation group according to TM perforation grade. The mean perforation closure times were $37.3 \pm 24.5$ in the approximation group and $36.4 \pm 26.4$ days in the ESM patch group in patients with small traumatic TM perforation. Complete closure time was not statistically different between the 2 groups $(p=0.205)$. However, in patients with moderate-to-large traumatic TM perforation, the mean perforation closure times was $110.4 \pm 127.4$ in the approximation group and $77.4 \pm 78.6$ days in the ESM patch group. Healing time was significantly shorter in the ESM patch group than in the approximation group in patients with moderate-to-large traumatic TM perforation $\left(p=0.007^{*}\right)$. *statistically significant. ESM: eggshell membrane, TM: tympanic membrane. 


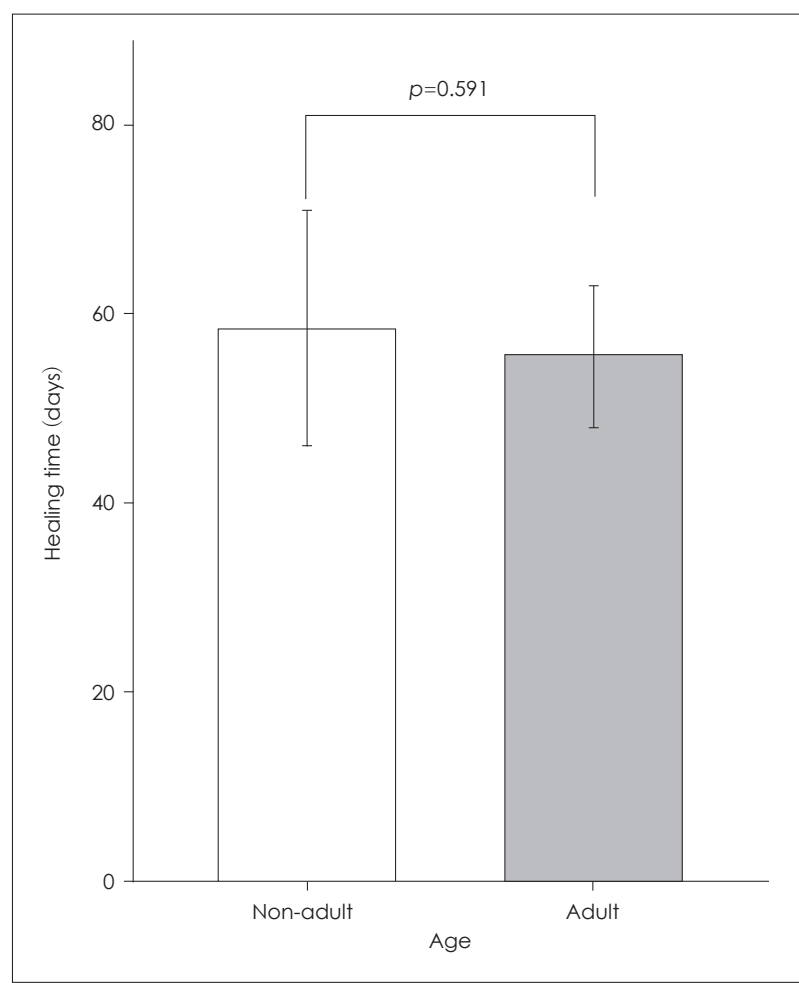

Fig. 3. Healing time in ESM patching patients according to patient age group. The mean healing time was $58.5 \pm 23.9$ days in nonadult patients ( $\leq 18 \mathrm{yrs}$ ) and $56.4 \pm 14.7$ days in adult patients $(>18$ yrs). This result was not significant $(p=0.591)$. ESM: eggshell membrane.

pared with an edge-approximated group for TM perforation, and we reported that an ESM patch did not affect healing rate [10]. However healing time was shorter in the patch application group [10]. The present study included almost 4 times more patients, affording results that are more definitive. In addition, we conducted further research in to the relationship between healing time and Griffin's grade, age, patching time, and perforation location.

Various materials are available for TM perforation patch application, including gentamicin ointment plugs, paper, tape, silk, water-soluble chitosan, alloderm, urinary bladder matrix, and growth factor combined patches [13-17]. ESM patches have several advantages over the use of other materials, including anti-infection properties, water insolubility, similar physical properties to the TM for sound conduction, and low cost [18-20].

In the current study, we show that ESM patching reduces healing time compared with edge approximation only, especially in patients with moderate or large traumatic TM perforation ( $\geq$ grade II). Small TM perforations have high healing rates and short closure times, without any intervention [12].

Our study also showed that there was no significant difference between early patching (less than 1 week after injury)

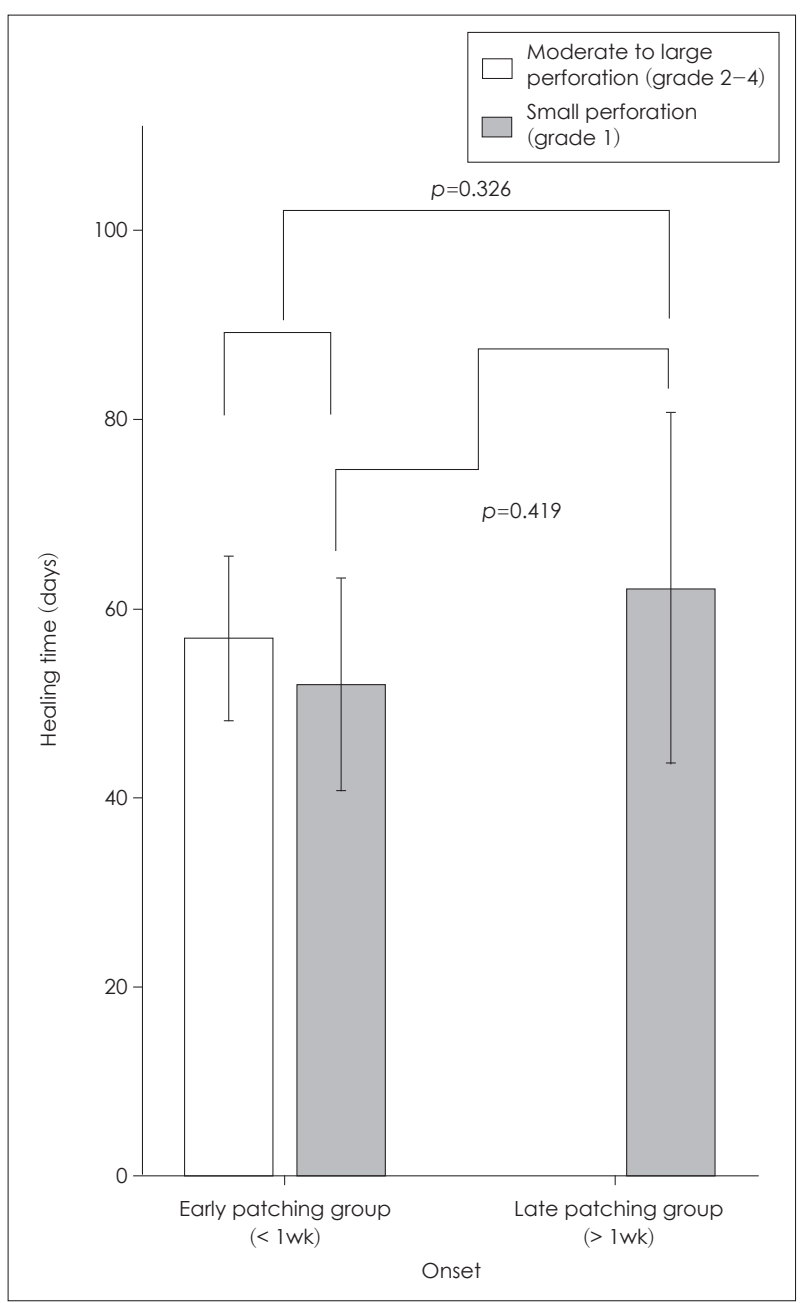

Fig. 4. Healing time in early patching group and late patching group according to TM perforation grade. The mean TM closure time was $54.8 \pm 34.7$ days for early patching patients ( $<1$ week post injury) and $62.3 \pm 39.6$ days for late patching patients ( $\geq 1$ week post injury, $p=0.326$ ). In the small TM perforation patients, there was no significant difference in healing time between the early patching group and the late patching group $(p=0.419)$. TM: tympanic membrane.

and late patching (more than 1 week after injury) in patients with ESM patching group. We assume that late patching has a similar effect on healing time. However, no patients with moderate or large TM perforations received ESM patching more than 1 week after injury, therefore further studies are required in this patient group. In addition, healing time was not affected by age or perforation location.

In conclusion, ESM patch application in patients with moderate or large traumatic TM perforation ( $\geq$ grade II) is beneficial for reducing healing time and avoiding infection at any time post-injury, at any age, or at any tympanic membrane injury site. 


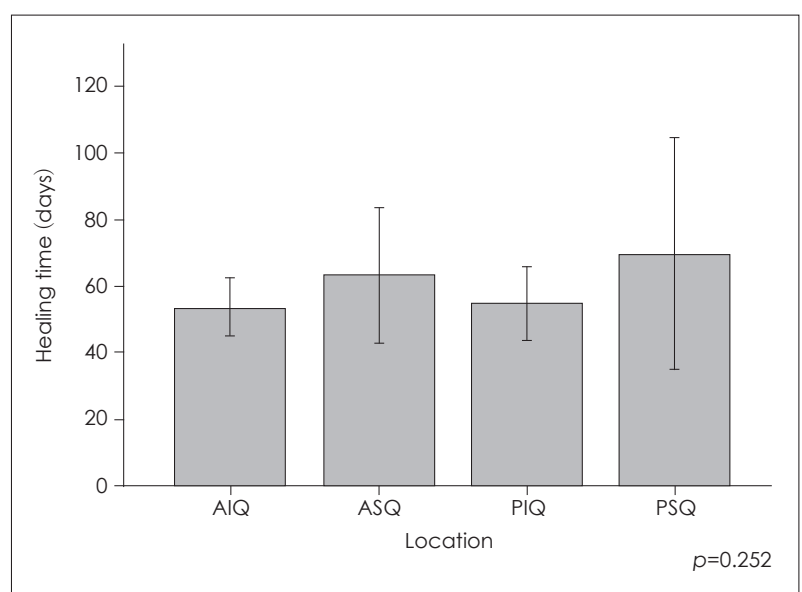

Fig. 5. Healing time according to location of TM perforation in ESM patching patients. The mean TM closure time was $54.9 \pm 21.6$ days for patients with AIQ TM perforation, $63.4 \pm 42.3$ days for patients with ASQ TM perforation, $55.3 \pm 24.2$ days for patients with PIQ TM perforation, and $70.2 \pm 76.5$ days for patients with PSQ TM perforation. There were no significant differences ( $p=0.252$, ANOVA). AIQ: anterior inferior quadrant, ASQ: anterior superior quadrant, $\mathrm{PIQ}$ : posterior inferior quadrant, $\mathrm{PSQ}$ : posterior superior quadrant, ESM: eggshell membrane, TM: tympanic membrane.

\section{Acknowledgments}

This work was supported by the Daewoong Pharmaceutical Co. LTD (AS10131-001).

\section{Conflicts of interest}

The authors have no financial conflicts of interest.

\section{REFERENCES}

1) Saito H, Kazama Y, Yazawa Y. Simple maneuver for closing traumatic eardrum perforation by micropore strip tape patching. Am J Otol 1990;11:427-30.

2) Lasak JM, Van Ess M, Kryzer TC, Cummings RJ. Middle ear injury through the external auditory canal: a review of 44 cases. Ear Nose Throat J 2006;85:722, 724-8.

3) Ritenour AE, Wickley A, Ritenour JS, Kriete BR, Blackbourne LH, Holcomb JB, et al. Tympanic membrane perforation and hearing loss from blast overpressure in Operation Enduring Freedom and Operation Iraqi Freedom wounded. J Trauma 2008;64:S174-8; discussion S8.

4) Hempel JM, Becker A, Müller J, Krause E, Berghaus A, Braun T. Traumatic tympanic membrane perforations: clinical and audio- metric findings in 198 patients. Otol Neurotol 2012;33:1357-62.

5) Lou ZC, He JG. A randomised controlled trial comparing spontaneous healing, gelfoam patching and edge-approximation plus gelfoam patching in traumatic tympanic membrane perforation with inverted or everted edges. Clin Otolaryngol 2011;36:221-6.

6) Orji FT, Agu CC. Determinants of spontaneous healing in traumatic perforations of the tympanic membrane. Clin Otolaryngol 2008;33: 420-6.

7) Conoyer JM, Kaylie DM, Jackson CG. Otologic surgery following ear trauma. Otolaryngol Head Neck Surg 2007;137:757-61.

8) Sprem N, Branica S, Dawidowsky K. Tympanoplasty after war blast lesions of the eardrum: retrospective study. Croat Med J 2001; 42:642-5.

9) Camnitz PS, Bost WS. Traumatic perforations of the tympanic membrane: early closure with paper tape patching. Otolaryngol Head Neck Surg 1985;93:220-3.

10) Jun HJ, Oh KH, Yoo J, Han WG, Chang J, Jung HH, et al. A new patch material for tympanic membrane perforation by trauma: the membrane of a hen egg shell. Acta Otolaryngol 2014;134:250-4.

11) Griffin WL Jr. A retrospective study of traumatic tympanic membrane perforations in a clinical practice. Laryngoscope 1979;89:26182.

12) Lou ZC, Lou ZH, Zhang QP. Traumatic tympanic membrane perforations: a study of etiology and factors affecting outcome. Am J Otolaryngol 2012;33:549-55.

13) Kim J, Kim CH, Park CH, Seo JN, Kweon H, Kang SW, et al. Comparison of methods for the repair of acute tympanic membrane perforations: silk patch vs. paper patch. Wound Repair Regen 2010;18: 132-8.

14) Kim JH, Choi SJ, Park JS, Lim KT, Choung PH, Kim SW, et al. Tympanic membrane regeneration using a water-soluble chitosan patch. Tissue Eng Part A 2010;16:225-32.

15) Parekh A, Mantle B, Banks J, Swarts JD, Badylak SF, Dohar JE, et al. Repair of the tympanic membrane with urinary bladder matrix. Laryngoscope 2009;119:1206-13.

16) Hakuba N, Iwanaga M, Tanaka S, Hiratsuka Y, Kumabe Y, Konishi $\mathrm{M}$, et al. Basic fibroblast growth factor combined with atelocollagen for closing chronic tympanic membrane perforations in 87 patients. Otol Neurotol 2010;31:118-21.

17) Golz A, Goldenberg D, Netzer A, Fradis M, Westerman ST, Westerman LM, et al. Paper patching for chronic tympanic membrane perforations. Otolaryngol Head Neck Surg 2003;128:565-70.

18) Lim DJ. Human tympanic membrane. An ultrastructural observation. Acta Otolaryngol 1970;70:176-86.

19) Cheng T, Dai C, Gan RZ. Viscoelastic properties of human tympanic membrane. Ann Biomed Eng 2007;35:305-14.

20) Torres FG, Troncoso OP, Piaggio F, Hijar A. Structure-property relationships of a biopolymer network: the eggshell membrane. Acta Biomater 2010;6:3687-93 